\title{
Improving documentation of physical health investigations in an adolescent mental health inpatient unit
}

\author{
David Horton \\ Oxford Health NHS Foundation Trust, UK
}

\begin{abstract}
Physical health investigations, such as blood tests, ECGs, and appropriate radiological tests, are essential in the assessment and management of many patients in inpatient mental health settings. This project took place in a 12-bed adolescent mental health unit in Swindon, UK, where on average at least two-thirds of patients have a diagnosed eating disorder. Multidisciplinary ward rounds provide an appropriate setting for discussion and documentation of physical investigations. Over a two-week period, 22 electronic ward round entries were audited for any documentation of five common investigations - blood tests, ECG, MRI head, DEXA, and ovarian ultrasound. Blood tests were documented in 2/22 (9.1\%), ECG, MRI head, DEXA, and ovarian ultrasound were documented in 0/22 (0\%). Modifications were made to an electronic ward round template, to include headings for each of these investigations, with free-text boxes as well as drop-down boxes for the radiological tests. Following this, re-audit of 22 ward round entries over a two-week period showed documentation had hugely improved blood tests were documented in 21/22 (95.5\%), with ECG, MRI head, DEXA, and pelvis US all documented in 22/22 (100\%). A further audit a month later showed these results were largely sustained. In conclusion, use of a simple, structured ward round template can hugely improve documentation of important physical investigations within mental health settings.
\end{abstract}

\section{Problem}

Physical health investigations are often not appropriately performed or documented in mental health patients, including in children and adolescent units [1]. There has been increasing recognition of the importance of intervening to improve physical health in psychiatric inpatients in general [2]. Physical health monitoring of such patients is vital in informing the assessment and management of patients with psychiatric illness, as well as in monitoring medication in these patients.

This project took place at Marlborough House adolescent inpatient unit in Swindon, UK, which is a 12-bed adolescent psychiatric ward. Prior to this project, blood test results were recorded on paper and stored in a blood results folder. This was accessible to the medical team in the ward office. However, these results were not uploaded to the electronic patient notes system. As a result, some blood tests might have been performed and results obtained, but these would not be documented in the electronic notes. There was no set procedure for the documentation of other physical health investigations. Therefore, unless specifically brought up by a member of the medical team for discussion, other potentially relevant physical investigations were not routinely discussed or documented for each individual patient.

Documentation of physical investigations in patient notes is important in allowing all members of the multidisciplinary team to access them when needed, in helping to ensure results are taken into account when making clinical decisions, and in order to follow professional guidance on recording of clinical information. The aim of this project was to improve documentation of both the consideration of whether a particular investigation was indicated or not, and the documentation of results of already completed investigations.

\section{Background}

Marlborough House is a 12-bed Tier 4 adolescent mental health unit in Swindon, UK. At any time, approximately two-thirds of patients are likely to have a diagnosis of an eating disorder, while other patients have conditions including psychotic disorders, affective disorders, anxiety disorders, and emerging personality disorders. Patients with mental health disorders are known to be more likely to present with physical health problems and complications. All newly admitted patients would routinely have blood tests and an ECG on admission. Patients with eating disorders such as anorexia nervosa would normally require at least weekly blood tests, ECG monitoring, and consideration of investigations such as a DEXA scan to evaluate bone health and ovarian ultrasound to evaluate amenorrhoea. Patients with psychosis or atypical presentations may require an MRI head to exclude an organic cause.

At Marlborough House, ward rounds of patients under each of the two inpatient consultants occur once weekly. These are multidisciplinary meetings which determine the ongoing management plan. This is the usual forum for the discussion of physical health investigations - whether this be results of investigations already performed such as blood tests, or the consideration of whether more specialist tests are indicated and required for each patient or not. The ward uses an electronic medical notes system, in common with many other mental health units. A pre-designed Microsoft Word template is used to record multidisciplinary feedback during ward rounds, which is completed by a junior doctor and then uploaded to the electronic medical notes. 


\section{Baseline measurement}

An audit was completed of the electronic notes system in April 2015 , looking at a two week period, to identify ward round documentation of five different physical investigations - blood tests, ECG, MRI head, DEXA and ovarian ultrasound. If any of these investigations were included within the ward round entry - whether in terms of them being discussed, requested, or the results of investigations already performed - they were counted as documented. Otherwise, they were counted as not documented.

Over a two-week period, eleven patients had a ward round each week, giving a total of 22 ward round entries to be included. Blood tests were documented in 2 of 22 entries (9.1\%). ECG, MRI head, DEXA and ovarian ultrasound investigations were documented in 0 of 22 entries ( $0 \%$ for each).

A further retrospective audit was carried out into the number of physical investigations which had actually been completed on these patients. Of the 12 patients, 8 had a diagnosed eating disorder. An audit of the number of patients who had blood tests performed and documented in the paper results folder found that 11 of 12 patients had had blood tests completed prior to the time of ward round entries reviewed in April 2015. The one patient who had not had bloods had refused. Therefore, in terms of documentation in the medical notes, results should have been documented in 20 of 22 entries $(90.5 \%)$, but as stated above was only documented in 2 of 22 entries (9.1\%).

An audit of the number of ECGs performed but not documented in the notes was not possible for practical reasons, as a number of patients had been discharged by the time of this audit meaning it was not reliably possible to locate paper ECGs for these patients. This further emphasised the importance of documentation in the electronic notes. Audit of the three radiological investigations showed that by the time of the reviewed ward rounds in April 2015, 2 patients had previously had an MRI head, 3 patients had previously had a DEXA and 2 patients had previously had an ultrasound pelvis. However, in addition to these investigations already performed, these investigations should have been discussed for the remaining eating disorder patients and a decision made over whether these would be indicated and useful.

In conclusion, baseline measurement confirmed there was a disparity between the number of investigations performed, with results available elsewhere, and the number being documented in ward rounds in the electronic notes. It also suggested that there was no documentation of consideration for these investigations in patients where they had not yet been performed, but might be indicated.

See supplementary file: ds5798.docx - "QIP - physical investigations baseline April 2015"

\section{Design}

After consulting with the two consultant psychiatrists, other junior doctors, nursing staff, and ward matron, it was felt that modifying the ward round template which was already in use would be the most apparent way to improve documentation of physical health investigations during ward rounds. This would serve as a prompt to discuss the appropriate investigations in ward round and to then document this, as well as allowing opportunity for results of completed investigations to be reviewed, discussed, and documented.

\section{Strategy}

PDSA Cycle 1

Initially discussions took place with the administration team in order to modify the ward round template. Initially, a section was added to the medical feedback section of the template with five headings: "Bloods:", "ECG", MRI Head", "DEXA", "Ovarian US". This was then shown to the other doctors on the ward and trialled in one ward round. Whilst agreeing with the principle, it was felt that free-text boxes, particularly for MRI, DEXA, and ovarian US, might be left blank as they are not relevant to every patient, which would not improve the problem of poor documentation.

PDSA Cycle 2

Further discussions took place with the administration team, and drop-down boxes were then added to each of the headings for "MRI Head", "DEXA" and "Ovarian US". There were three options "Required", "Not Required", or "Done Previously". There was no option to leave these blank. There were also free-text boxes adjacent to these drop-down boxes so that results or other information could be entered if appropriate. The sections for "Bloods" and "ECG" remained free-text boxes as these are required for all patients on admission. This was agreed by all doctors on the ward to be an efficient and stringent way to improve documentation, and it was agreed this would serve as a prompt to ensure discussion about these tests during ward rounds. The template was then introduced to be used in all future ward rounds.

\section{Results}

A further audit of the electronic notes system took place in June 2015, after approximately one month of the new template being used. This took place over a two-week period, with 10 patients having a ward round each week, and a further 2 patients having one ward round during this period, giving a total of 22 ward round entries. The same five investigations were included. If any of these investigations were included within the ward round entry - whether in terms of them being discussed, requested, or results of investigations already performed - they were counted as documented. Otherwise, they were counted as not documented. Blood tests were documented in 21 of 22 entries (95.5\%). ECG, MRI head, DEXA, and pelvic ultrasound was documented in 22 of 22 entries (100\% for each).

A final audit, using the same methodology and comparing the same investigations, was conducted over a two-week period in July 2015 
in order to establish whether the improvement was sustained. Of a total of 22 ward round entries, blood tests were documented in 20 of 22 entries (90.1\%), ECG in 21 of 22 entries (95.5\%), with MRI head, DEXA, and pelvic ultrasound documented in 22 of 22 entries (100\% for each).

See supplementary file: ds5797.docx - "QIP - results graph"

\section{Lessons and limitations}

This project demonstrated that small changes can yield significant improvements. Ward rounds can be rushed due to the time pressures and clinical workload of the team, and at times documentation might reflect this, with missing or incomplete items. In this case, using a simple structured template, with a mixture of free-text and drop-down boxes, served as a prompt for discussion and documentation of these important investigations. It was important to utilise the knowledge of the administration team who were able to make helpful suggestions for how to improve the template and its documentation in the notes.

One limitation in this study is that it has used an electronic template which allows drop-down boxes, which means these sections cannot be left blank. If paper templates were used, the appropriate options could be included to be circled or crossed out, but it is possible that this would not be utilised and these could still be left blank.

However, it would still be likely to serve as a prompt which would be expected to improve documentation compared to no intervention at all.

It is important to stress that the aim of this project was not to encourage investigations to be performed which were not necessary, but simply to improve documentation. Not all these physical investigations would be appropriate or possible for every patient, nevertheless a template such as this can act as a prompt to remind the mental health team to consider the usefulness of these investigations for each individual. It is possible that this could serve to increase the number of investigations carried out even though this was not the aim of the project.

\section{Conclusion}

A simple structured template has significantly improved the documentation of important physical investigations in this adolescent mental health ward. The change was highly significant from nearly no documentation at all to near-complete documentation following the intervention. The final audit in July showed these results were largely maintained over time. When working on a busy mental health ward with long and in-depth ward rounds, having prompts to discuss and document physical health investigations has proved very helpful in ensuring physical health needs are not forgotten or poorly documented. Many mental health units use electronic notes systems and have a similar style of multidisciplinary ward rounds with electronic documentation, which means this design would be transferable to many other settings.
1. Pasha N, Saeed S, Drewek K. Monitoring of physical health parameters for inpatients on a child and adolescent mental health unit receiving regular antipsychotic therapy. BMJ Qual Improv Report 2015;4:1.

2. Hjorth P, Davidsen AS, Kilian R et al. Improving the physical health of long-term psychiatric inpatients. Aust N Z J Psychiatry 2014; 48(9):861-70

\section{Declaration of interests}

Nothing to declare.

\section{Acknowledgements}

Dr Gillian Combe, Dr Richard Eyre, Dr Josie Lee, Amanda Cadder, Susan McKellar, Beris Cumming.

\section{Ethical approval}

Ethical approval was not deemed to be required, as documentation of relevant clinical information is a universally recommended practice and is included in the GMC's guidance on Good Medical Practice. Documentation within patient notes is routinely audited for many other measures for audit and regulatory purposes, for example in meeting specific CQC or Trust targets.

\section{References}

